\title{
EFECTO DEL CONSUMO DE CREATINA SOBRE LA EJECUCIÓN DE DESTREZAS REALIZADAS EN FORMA INTERMITENTE Y DE DURACIÓN PROLONGADA
}

\author{
Oscar Pablo Gutiérrez Sancho, José Moncada Jiménez, y Walter Salazar Rojas \\ Escuela de Educación Física y Deportes \\ Universidad de Costa Rica, San José, Costa Rica \\ E-mail: opgutier@costarricense.cr
}

\begin{abstract}
Resumen
Gutiérrez Sancho, O. P., Moncada Jiménez, J., y Salazar Rojas, W. (2002). Efecto del consumo de creatina sobre la ejecución de destrezas realizadas en forma intermitente y de duración prolongada. Revista de Ciencias del Ejercicio y la Salud, 2(1), 23-30. Se estudió el efecto de la suplementación con creatina (Cr) sobre el rendimiento en natación, ciclismo y atletismo en 10 mujeres. Las participantes debían realizar 4 intervalos en cada deporte a máxima intensidad, además de un segmento aeróbico de 7 min post intervalo entre el $65-85 \%$ de la $F C_{\text {máx. }}$ En un diseño de medidas repetidas, las atletas completaron 3 condiciones experimentales: control (CT), suplementación con creatina (CCr), y condición placebo (PL). Se registró el tiempo de cada intervalo en las diferentes condiciones y se aplicaron 3 ANOVAS de medidas repetidas para cada deporte. Se encontraron interacciones entre los tratamientos y las mediciones en ciclismo $(p=.001)$ y atletismo $(p=.002)$. El análisis de seguimiento para la interacción de ciclismo reveló que todas las condiciones fueron diferentes entre sí ( $p<0.05)$, y que éstas diferencias en los promedios se encontraban en los intervalos 1, 2 y 4. El análisis post-hoc de Tukey mostró diferencias $(p<0.05)$ en el intervalo 1 entre CT $(M=78.3 \pm 11.58 s)$ y $P L(M=75.2 \pm 7.62 s)(p<0.05)$. En el intervalo 2 las diferencias fueron entre $C T$ $(M=77.3 \pm 7.97 s)$ y $C C r(M=75.3 \pm 7.97 s)(p<0.05)$; y entre $C T(M=77.3 \pm 7.97 s)$ y $P L(M=74.8 \pm$ 7.28 s) $(p<0.05)$. En el intervalo 4 , $C C r(M=77.5 \pm 8.29$ s) y $P L(M=76.4 \pm 7.49$ s). El análisis de seguimiento de la interacción de atletismo mostró diferencias en los intervalos 1, 3 y 4. El análisis post-hoc de Tukey indicó diferencias en el intervalo 1 de atletismo entre $\operatorname{CCr}(M=96.6 \pm 13.63$ s) y PL $(M=90.4 \pm$ $14.08 s)$ y entre $C C r(M=96.6 \pm 13.63 s)$ y $C T(M=91.8 \pm 9.73 s)(p<0.05)$. En el intervalo 3 hubo diferencias entre CT $(M=94.8 \pm 13.60 \mathrm{~s})$ y $P L(M=90.2 \pm 8.97 \mathrm{~s})$. Por último, en el intervalo 4 las diferencias fueron entre CT $(M=96.9 \pm 15.18 \mathrm{~s})$ y $C C r(M=90.3 \pm 11.84 \mathrm{~s}) ;$ y entre $C T(M=96.9 \pm 15.18$ s) y PL $(M=90.7 \pm 11.35 \mathrm{~s})(p<0.05)$. Se concluye que durante las etapas de atletismo hubo un efecto positivo de la suplementación con $\mathrm{Cr}$ y del placebo. PALABRAS CLAVES: Creatina, triatlón, ayudas ergogénicas, placebo.
\end{abstract}

\section{INTRODUCCIÓN}

Según Lamb (1999), el sistema energético más rápido que dispone el cuerpo para resintetizar ATP en el músculo, es el fosfato de creatina ( $\mathrm{PCr}$ ). Esta reacción química, catalizada por la enzima creatina fosfoquinasa $(\mathrm{CK})$, transfiere de forma directa el fosfato que porta el PCr al ADP, lo anterior apoyado en la revisión del American College of Sports Medicine (ACSM, 2000). La creatina $(\mathrm{Cr})$ a su vez, es una proteína fisiológicamente activa, necesaria para formar $\mathrm{PCr}$ en el músculo. Esta fuente energética, es indispensable durante el ejercicio intenso (90- $\left.100 \% \mathrm{VO}_{2 \text { máx }}\right)$ y de corta duración $(<1 \mathrm{~min})$ (Engelhardt, Neumann, Berbalk, y Reuter, 1998; Greenhaff, Bodin, Soderlund, y Hultman, 1994; Juhn, 1999). En diferentes estudios se ha reportado que el consumo de $\mathrm{Cr}$ podría mejorar el desempeño físico, principalmente durante ejercicios anaeróbicos repetitivos (Greenhaff, 1995; Kamber, Koster, Kreis, Walker, Boesch, y Hoppeler, 1999; Kreider, Ferreira, Wilson, Grindstaff, Plisk, Reinardy, Cantler, y Almada, 1998; Preen, Dawson, Goodman, Lawrence, Beilby, y Ching, 2001), más no en todos se ha encontrado beneficio (Burke, Pyne, y Telford, 1996; Green, McLester, Smith, y Mansfield, 
2001; Snow, McKenna, Kemp, Stathis, y Zhao, 1998).

Durante el ejercicio de alta intensidad, la aparición de la fatiga o la incapacidad para poder sostener el nivel de tensión muscular deseado, se ha relacionado con el agotamiento de las fuentes del sustrato energético PCr (Balsom, Söderlund, Sjödin, y Ekblom, 1995; Greenhaff et al., 1994; Harris, Viru, Greenhaff, y Hultman, 1992). Bajo este contexto es que nace el interés por el uso de la Cr y a la vez la postulación de teorías que explican los posibles mecanismos de cómo la suplementación exógena de $\mathrm{Cr}$ mejoraría el rendimiento en el ejercicio intenso e intermitente. En varios estudios se ha demostrado de forma consistente que el uso de ciertas dosis de $\mathrm{Cr}\left(2-30 \mathrm{gr} \cdot \mathrm{d}^{-1}\right)$, por periodos cortos (3-7 d) aumentan significativamente los valores totales de $\mathrm{Cr}$ $(\mathrm{PCr}+\mathrm{Cr})$ en el músculo (Balsom et al., 1995; Green, Hultman, Macdonald, Sewell, y Greenhaff, 1996; Greenhaff et al., 1994; Kreis, Kamber, Koster, Felblinger, Slotboom, Hoppeler, y Boesch, 1999; Preen et al., 2001; Rockwell, Walberg-Rankin, y Toderico, 2001; Snow et al., 1998: Volek et al., 1999). En algunos de estos estudios, se han encontrado relaciones directas entre los niveles de $\mathrm{Cr}$ y la reposición o resíntesis del $\mathrm{PCr}$ durante los descansos, luego de un ejercicio intermitente y máximo (Greenhaff et al., 1994; Smith, Montain, Matott, Zientara, Jolesz, y Fielding, 1998).

Lo anterior ha sido utilizado para explicar las diferencias en cuanto a un mejor desempeño físico reportado en los grupos o condiciones experimentales de $\mathrm{Cr}$ a diferencia de los resultados obtenidos con control o placebo (PL) (Greenhaff et al., 1994; Green et al., 1996). Se especula que la mejoría en la resíntesis, permite estar en mejores condiciones energéticas para la próxima repetición, y además se lograría retardar la aparición de la fatiga, al evitar la acumulación de ADP y la concentración elevada de ácido láctico (ACSM, 2000).

Existe una gran variabilidad en los resultados de los estudios con respecto al tipo de destreza deportiva que se utiliza para determinar los efectos ergogénicos de la $\mathrm{Cr}$
(ACSM, 2000). Se han diseñado experimentos en natación (Burke et al., 1996; Grindstaff et al., 1997; Peyrebrune, Nevill, Donaldson, y Cosford, 1998), atletismo en pista (Aaserund, Gramvik, Olsen, y Jensen, 1998; Redondo, Dowling, Graham, Almada, y Williams, 1996), ciclismo estático (Prevost, Nelson, y Morris, 1997; Rockwell et al., 2001), remos (Rossiter, Cannell, y Jakeman, 1996) y kayak (McNaughton, Dalton y Tarr, 1998). Los resultados de los diversos estudios han sido mixtos; sin embargo, hay una serie de investigaciones en las que se demostraron efectos ergogénicos positivos de la ingesta de $\mathrm{Cr}$ sobre el tiempo de ejecución de determinada destreza física (Aaserud et al., 1998; Grindstaff et al., 1997; Peyrebrune et al., 1998). Por ejemplo, se han encontrado resultados positivos en el ejercicio intenso (90-100\% $\mathrm{VO}_{2 \text { máx }}$, ejecutado en más de 3 ocasiones. Juhn (1999), propone en su revisión de literatura, que el tipo de repetición intensa o "sprint" (natación, carrera, ciclismo, etc.) requiere de más estudio y a la vez crear diseños experimentales que simulen las situaciones reales de la destreza, dado que la mayoría de los experimentos se han llevado a cabo en condiciones de laboratorio en cicloergómetro. También propone que el número de repeticiones intensas sea una variable a considerar, porque la evidencia aunque es conflictiva apunta más hacia el ejercicio repetitivo, lo cual concuerda con otras revisiones bibliográficas respecto al tema (ACSM, 2000; Greenhaff, 1995; Poortmans y Francaux, 2000).

Existen dos estudios que han examinado el efecto de la suplementación con $\mathrm{Cr}$ en el rendimiento en ejercicio máximo y repetitivo; pero con diferencias en la modalidad de ejercicio. Sin embargo ambos comparten una característica que no poseen otros estudios, la cual es haber llevado a los sujetos a una fatiga extrema (ejercicio acumulado $>1 \mathrm{~h}$ ). Por una parte, Engenlhardt y colaboradores (1998), en un diseño de medidas repetidas, demostraron que los triatlonistas que fueron suplementados con $\mathrm{Cr}$ (6 días), lograron realizar una mayor cantidad de intervalos a una alta intensidad en cicloergómetro en comparación con la 
condición PL. La característica primordial en el diseño fue que el ejercicio mixto (aeróbico $\mathrm{y}$ anaeróbico) sumaba un tiempo total $>1 \mathrm{~h}$, con lo cual se demostró que puede haber un efecto ergogénico en pruebas que alternan la intensidad del ejercicio por periodos prolongados de tiempo, lo cual podría aplicarse a una amplia gama de deportes como el triatlón o el duatlón, que por sus estrategias de competición necesitan realizar segmentos sumamente intensos de forma repetitiva.

Por otra parte, en el caso del estudio de Preen et al. (2001), una dosis de $20 \mathrm{gr} \cdot \mathrm{d}^{-1}$ - $5 \mathrm{~d}^{-1}$, mejoró el trabajo total y además hubo cambios significativos en la potencia pico, a lo largo de muchas series de ejercicio máximo en cicloergómetro. La característica principal en el estudio Preen et al., (2001), fue que el ejercicio total se prolongó por aproximadamente $80 \mathrm{~min}$, existiendo mejorías en el rendimiento durante las fases finales del mismo. El diseño del estudio fue de medidas repetidas $y$ aunque no contemplaba la fase aeróbica como tal, los descansos tenían un carácter submáximo y repetitivo.

Como lo mencionó Junh (1999), es necesario estudiar el efecto de la $\mathrm{Cr}$ en el tipo de sprint y su interacción en pruebas de campo. Para alcanzar la propuesta de Juhn (1999) es necesario un diseño experimental que examine el efecto de la suplementación con $\mathrm{Cr}$ sobre la ejecución de varias destrezas en forma simultánea. Por otra parte, el diseño experimental debería contemplar la duración total y la modalidad de ejercicio (aeróbico, anaeróbico), debido que otros autores reportan efectos de la $\mathrm{Cr}$ sobre el rendimiento cuando se alterna la intensidad del ejercicio (Engenlhardt et al., 1998; Preen et al., 2001).

Por lo anterior, el propósito de esta investigación fue conocer si la suplementación con $\mathrm{Cr}$ mejora el tiempo en varios intervalos máximos (sprints) realizados en diferentes destrezas (natación, ciclismo, y carrera pedestre) y alternando la intensidad de ejercicio (aeróbico, anaeróbico).

\section{METODOLOGÍA}

\section{Participantes}

En el estudio participaron mujeres integrantes de un equipo local de triatlón $(n=10)$. La edad promedio de las participantes fue 17. $6 \pm 1.6$ años, con una talla y un peso promedio de $156.3 \pm 4.6 \mathrm{~cm}$, y $56.9 \pm 6.5 \mathrm{~kg}$, respectivamente.

Cada una de las atletas se sometió a una revisión médica para determinar su estado general de salud. Además se les pidió a los padres y a las atletas que firmaran un formulario de consentimiento informado para participar en el estudio, que aunque no especificaba el tipo de sustancia y los posibles efectos ergogénicos, se les mencionó que no era una sustancia doping y que está aprobada y catalogada por la Administración de Drogas y Alimentos de los Estados Unidos (FDA), como un suplemento nutricional.

\section{Instrumentos de medición}

Cada participante utilizó un monitor de ritmo cardiaco marca Polar ${ }^{\mathbb{B} .}$ Para medir el tiempo se utilizaron 11 cronómetros marca Timex $^{\circledR}$, con capacidad para registrar $y$ almacenar hasta 100 lapsos cada uno. El peso corporal $(\mathrm{kg})$ se registró con una balanza electrónica y las salidas para cada sprint se dieron con una pistola de aire.

\section{Procedimientos}

Para describir mejor los procedimientos utilizados en este estudio, se presentan a continuación dos secciones: a) diseño experimental; y b) suplementación.

Diseño experimental. En un diseño contrabalanceado, las participantes pasaron por tres condiciones experimentales: a) control (CT); b) suplementación con creatina $(\mathrm{CCr})$, y c) suplementación con placebo (PL). Cada condición estuvo separada por 20 días, y las participantes debían cumplir con el mismo protocolo experimental, que se mantuvo constante para todas las condiciones. Este se describe en el siguiente 
párrafo, pero en resumen consistía en realizar un sprint al $100 \%$ de intensidad, seguido de 7 min de ejercicio al $65-85 \%$ de la $\mathrm{FC}_{\text {máx }}$.

Las participantes fueron citadas a las 7 a.m. al complejo deportivo donde acostumbraban entrenar. Posteriormente realizaron un calentamiento breve de acuerdo con sus hábitos, pero sin incluir ejercicios intensos o prolongados. Seguidamente las atletas debían realizar 4 sprints de natación $(100 \mathrm{~m}), 4$ sprints de ciclismo $(600 \mathrm{~m})$ y 4 sprints de atletismo $(400 \mathrm{~m})$. Las distancias en los sprints fueron asignadas con base a un estudio piloto, donde se ajusto la distancia con respecto duración por deporte, tratando de equiparar el tiempo de ejercicio en todos los sprints. Entre cada sprint realizaron $7 \mathrm{~min}$ de ejercicio aeróbico (65-85\% $\left.\quad \mathrm{FC}_{\text {máx }}\right)$ del mismo deporte, controlando la FC con monitor Polar $^{\circledR}$ a intervalos de $1 \mathrm{~min}$. No hubo descansos totales hasta terminar el diseño experimental de ese día particular, excepto el tiempo de transición que transcurre de un deporte a otro. Tres días antes a la prueba, las atletas no realizaron ningún tipo de entrenamiento de velocidad y un día antes no realizaron ejercicio físico del todo. La cena del día anterior a la prueba fue equiparada en contenido energético. El peso se controló para descartar un estado de deshidratación antes del ejercicio. Además, se permitió la ingesta de agua en forma voluntaria, antes y durante la prueba.

Suplementación. Durante 7 días, las participantes recibieron dos tipos de suplementación. La suplementación con cretina ( $\mathrm{CCr}$ ), consistió en la ingesta de $0.25 \mathrm{gr} \cdot \mathrm{kg} \cdot \mathrm{d}$ de monohidrato de creatina marca Labrada ${ }^{\circledR}$, dividido en 2 dosis diarias, una en la mañana y otra en la noche. Cada dosis se disolvió en 250 $\mathrm{ml}$ de jugo de uvas. Se seleccionó esta duración debido a que existe suficiente evidencia que demuestra que periodos cortos de suplementación con $\mathrm{Cr}(\leq 5$ días) produce un aumento en los niveles totales de $\mathrm{Cr}$ y a la vez aumenta la expulsión de creatinina, tanto en hombres como en mujeres (Casey, ConstantinTeodosiu, Howell, Hultman, y Greenhaff, 1996; Green et al., 1996; Kamber et al., 1999).

La condición PL consistió en suministrar maltodextrinas en igual dosis a la $\mathrm{CCr}$, disueltas también en jugo de uvas. La mayoría de los diseños experimentales sobre los efectos de la $\mathrm{Cr}$ en el rendimiento anaeróbico, han utilizado carbohidratos (maltodextrinas) para los grupos o condiciones PL (Poortmans et al., 2000 ).

\section{Análisis Estadístico}

Para analizar los datos se utilizó el Paquete Estadístico para las Ciencias Sociales (SPSS, 1998). Los datos se presentan como promedio (M) y la desviación estándar $( \pm D S)$. Se utilizaron análisis de varianza (ANOVA) de dos vías de medidas repetidas en ambos factores ( 3 condiciones x 4 sprints) para cada deporte. Cuando hubo interacciones significativas, se procedió con los análisis de efectos simples, según correspondía, seguidamente del análisis post hoc de Tukey. Finalmente, se calculó la varianza explicada por medio del estadístico omega al cuadrado $\left(\omega^{2}\right)$.

\section{RESULTADOS}

Las pruebas de ANOVA para medidas repetidas revelaron dos interacciones significativas $(p<0.05)$, para ciclismo $\left(\omega^{2}=\right.$ $11.24 \%)$ y para atletismo $\left(\omega^{2}=10.59 \%\right)$, mientras que no hubo una interacción significativa en natación $(p=0.725)$ (Tabla $1)$. 
Tabla 1. Tiempos en los sprints de natación $(4 \times 100 \mathrm{~m})$. Los valores representan el promedio $(M)$ y la desviación estándar $( \pm D S)$.

\begin{tabular}{lrrrr}
\hline & \multicolumn{4}{c}{ Número de sprint y tiempo realizado (s) } \\
\cline { 2 - 5 } Condición experimental & $\mathbf{1}$ & $\mathbf{2}$ & $\mathbf{3}$ & \multicolumn{1}{c}{$\mathbf{4}$} \\
\hline Control & $99 \pm 19.26$ & $100.60 \pm 18.99$ & $101.4 \pm 18.60$ & $103.7 \pm 18.50$ \\
Con Creatina & $92.6 \pm 14.37$ & $96.2 \pm 16.72$ & $96.9 \pm 16.99$ & $98.5 \pm 16.62$ \\
Placebo & $93.9 \pm 18.28$ & $95.1 \pm 16.11$ & $97.6 \pm 15.92$ & $100 \pm 15.70$ \\
\hline
\end{tabular}

El análisis de efectos simples para ciclismo, reveló que las 3 condiciones experimentales eran diferentes entre sí $(p<$ 0.05) (Tabla 2). Un segundo análisis de seguimiento mostró que las diferencias significativas se encontraban en los intervalos 1,2 y $4(p<0.05)$. Los análisis post hoc de Tukey revelaron diferencias en el intervalo 1 de ciclismo entre CT $(M=78.3 \pm 11.58 \mathrm{~s})$ y PL $(M=75.2 \pm 7.62 \mathrm{~s})(p<0.05)$. En el intervalo 2 , hubo diferencias entre CT $(M=$ $77.3 \pm 7.97 \mathrm{~s})$ y $\operatorname{CCr}(M=75.3 \pm 7.97 \mathrm{~s})(p<$ $0.05)$; y entre CT $(M=77.3 \pm 7.97 \mathrm{~s})$ y PL $(M$ $=74.8 \pm 7.28 \mathrm{~s})(p<0.05)$. Finalmente, en el intervalo 4, las diferencias fueron entre $\mathrm{CCr}$ $(M=77.5 \pm 8.29 \mathrm{~s})$ y $\mathrm{PL}(M=76.4 \pm 7.49 \mathrm{~s})$ $(p<0.05)$.

Por otra parte, el análisis de efectos simples para atletismo, reveló que existían diferencias significativas $(p<0.05)$ entre todas las condiciones experimentales (Tabla 3). El análisis de seguimiento reveló diferencias significativas $(p<0.05)$ en los intervalos 1, 3 y 4 . El análisis post hoc de Tukey indicó diferencias en el intervalo 1 entre $\operatorname{CCr}(M=96.6 \pm 13.63 \mathrm{~s})$ y $\mathrm{PL}(M=$ $90.4 \pm 14.08 \mathrm{~s})(p<0.05) ; \mathrm{y}$ entre $\operatorname{CCr}(M=$ $96.6 \pm 13.63 \mathrm{~s})$ у CT $(M=91.8 \pm 9.73 \mathrm{~s})(p<$ $0.05)$. En el intervalo 3, las diferencias fueron entre CT $(M=94.8 \pm 13.60 \mathrm{~s})$ y PL $(M=$
$90.2 \pm 8.97 \mathrm{~s})(p<0.05)$. Finalmente, en el caso del intervalo 4, las diferencias fueron entre CT $(M=96.9 \pm 15.18 \mathrm{~s})$ y $\mathrm{CCr}(M=$ $90.3 \pm 11.84 \mathrm{~s}) ; \mathrm{y}$ entre CT $(M=96.9 \pm 15.18$ s) y PL ( $M=90.7 \pm 11.35 \mathrm{~s})$.

\section{DISCUSIÓN}

El propósito de este estudio era demostrar si la suplementación con Cr lograba mejorar el rendimiento en tres diferentes destrezas, natación, ciclismo, y atletismo; ejecutadas de forma mixta (anaeróbica - aeróbica) y prolongada $(>1 \mathrm{~h})$.

Existen estudios en los que se ha demostrado un efecto positivo de la suplementación con $\mathrm{Cr}$ en el rendimiento durante ejercicios intermitentes y prolongados (Engenlhardt et al, 1998; Preen et al, 2001). A pesar de ello, en el presente estudio se muestran resultados mixtos; por una parte, al descomponer las interacciones se observa que la suplementación con $\mathrm{Cr}$ ocasiona una mejoría en el rendimiento en los intervalos finales del atletismo (Gráfico 1); no obstante, también hay una tendencia a que el rendimiento permanezca estable en la condición PL (Gráfico 1).

Tabla 2. Tiempos en los sprints de ciclismo $(4 \times 600 \mathrm{~m})$. Los valores representan el promedio $(M)$ y la desviación estándar ( $\pm D S)$.

\begin{tabular}{lcccc}
\hline & \multicolumn{4}{c}{ Número de sprint y tiempo realizado (s) } \\
\cline { 2 - 5 } Condición experimental & $\mathbf{1}$ & $\mathbf{2}$ & $\mathbf{3}$ & $\mathbf{4}$ \\
\hline Control & $78.3 \pm 11.58$ & $77.3 \pm 7.97^{\mathrm{b}}$ & $76.6 \pm 9.49$ & $77.5 \pm 8.29$ \\
Con Creatina & $76.8 \pm 8.47$ & $75.3 \pm 7.97^{\mathrm{a}}$ & $76.7 \pm 7.75$ & $78.9 \pm 9.41^{\mathrm{b}}$ \\
Placebo & $75.2 \pm 7.66^{\mathrm{a}}$ & $74.8 \pm 7.28$ & $77.4 \pm 9.67$ & $76.4 \pm 7.49$ \\
\hline
\end{tabular}

${ }^{\mathrm{a}} p<.05$, diferente de la condición control

${ }^{\mathrm{b}} p<.05$, diferente de la condición placebo 
Tabla 3. Tiempos en los sprints de atletismo $(4 \times 400 \mathrm{~m})$. Los valores representan el promedio $(M)$ y la desviación estándar ( $\pm D S$ ).

\begin{tabular}{llccc}
\hline & \multicolumn{4}{c}{ Número de sprint y tiempo realizado (s) } \\
\cline { 2 - 5 } Condición experimental & \multicolumn{1}{c}{$\mathbf{1}$} & $\mathbf{2}$ & $\mathbf{3}$ \\
\hline Control & $91.8 \pm 9.73$ & $91.5 \pm 10.78$ & $94.8 \pm 13.60^{\mathrm{b}}$ & $96.9 \pm 15.18^{\mathrm{b}}$ \\
Con Creatina & $96.6 \pm 13.63^{\mathrm{a}} \mathrm{b}$ & $92.1 \pm 12.39$ & $93.7 \pm 8.47$ & $90.3 \pm 11.84^{\mathrm{a}}$ \\
Placebo & $90.4 \pm 14.08$ & $90.4 \pm 12.36$ & $90.2 \pm 8.97$ & $90.7 \pm 11.35$ \\
\hline
\end{tabular}

${ }^{\mathrm{a}} p<.05$, diferente de la condición control

${ }^{\mathrm{b}} p<.05$, diferente de la condición placebo

Parece que el efecto de la $\mathrm{Cr}$ se presenta en las fases finales del ejercicio $(>1$ h) así como lo han encontrado otros investigadores (Engenlhardt et al, 1998; Preen et al, 2001), no obstante también han observado mejorías en fases intermedias del ejercicio, lo cual no ocurrió en el presente estudio.

La explicación que más apoyo ha tenido en la literatura científica manifiesta que el efecto de la $\mathrm{Cr}$ está en mejorar la resíntesis de la $\mathrm{PCr}$ durante los descansos, para disponer de más energía en el ejercicio (Greenhaff et al.,1994; Smith et al., 1998; ACSM, 2000). Es posible que esta teoría pueda explicar los resultados de este estudio, dadas las características del diseño experimental a que fueron sometidas las atletas; en otras palabras, una serie de intervalos repetitivos de máxima intensidad con fases de recuperación aeróbicas submáximas.

Parece importante tomar en cuenta en futuros estudios la cantidad de ejercicio a que se someten los sujetos para causar la fatiga, puesto que en este estudio se encontró efectos al finalizar la actividad física. Es recomendable después de analizar los datos que en próximos estudios los protocolos de ejercicio lleven a los sujetos a una mayor fatiga. Por ejemplo, en algunos estudios con $\mathrm{Cr}$ donde realizaron pocas repeticiones $(\leq 3)$ en natación y atletismo (Redondo et al., 1996; Burke et al., 1996) no encontraron efectos significativos sobre el rendimiento.

Gráfico 1. Cambio en el tiempo $(\Delta \mathrm{s})$ para las condiciones experimentales en 4 intervalos de natación $(100 \mathrm{~m})$, ciclismo $(600 \mathrm{~m})$ y atletismo $(400 \mathrm{~m})$.

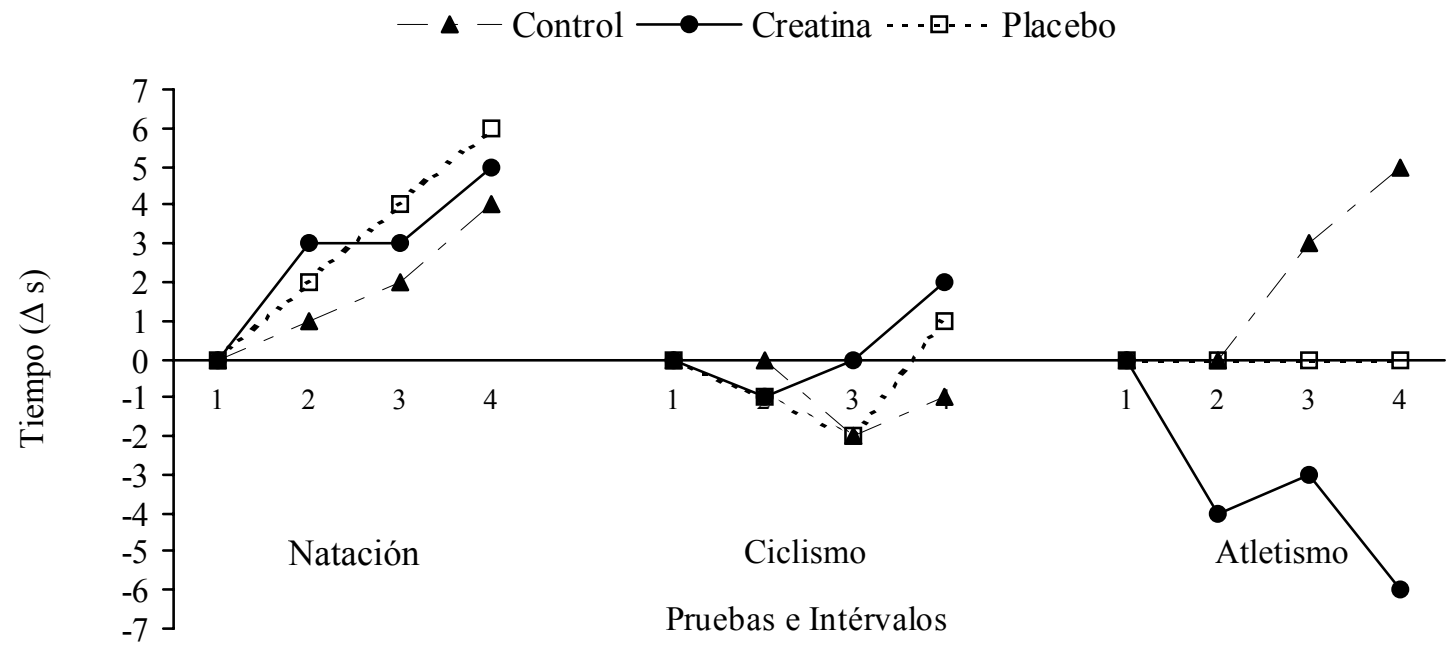


Es necesario aislar la disciplina de atletismo en el ejercicio intermitente $y$ prolongado, dado que en las destrezas de natación y ciclismo no parece haber resultados positivos del uso de Cr. Además, sería importante estudiar un posible efecto placebo en condiciones similares de ejercicio pero exclusivamente de atletismo, dado que hay evidencia de este efecto en el presente estudio. En otros estudios no han reportado un efecto placebo de la $\mathrm{Cr}$, sin embargo si hay ciertas tendencias del mismo (Balsom et al., 1995; Terrillion et al., 1997).

Para explicar por qué en la destreza de natación no hubo respuesta de la suplementación con $\mathrm{Cr}$, podría especularse que la cantidad de intervalos (4) en ese momento no era la necesaria para observar los efectos de la Cr. En otros estudios tampoco se han encontrado efectos en pruebas similares de natación con pocas repeticiones (Burke et al., 1996; Grindstaff et al., 1997).

Aunque la menstruación no fue controlada, no se observaron cambios importantes en el peso corporal de las participantes durante el estudio. Otras investigaciones en el tema de la suplementación con $\mathrm{Cr}$ podrían incorporar el ciclo menstrual como una variable de estudio; a pesar de que en una investigación reciente se refuta que durante las fases de la menstruación cambie la composición corporal y el rendimiento anaeróbico (Giacomoni, Bernard, Gavarry, Altare, y Falgairette, 2000).

La suplementación con $\mathrm{Cr}$ ha demostrando que puede optimizar variables relacionadas con el rendimiento físico como: potencia pico y trabajo total medidos en esfuerzos máximos de corta duración pero con muchos intervalos repetitivos que generan periodos largos de ejercicio acumulado ( $>1$ h) (Preen et al., 2001; Engenlhardt et al.,1998). No obstante no había evidencia de si era posible generalizar los resultados a otras disciplinas deportivas ejecutadas en forma simultanea y enfatizando en el cansancio en los sujetos.

En conclusión, en este estudio se ofrece evidencia de que la suplementación con $\mathrm{Cr}$ podría beneficiar el rendimiento anaeróbico en los segmentos finales de una competencia o entrenamiento de Triatlón o Duatlón (Gráfico 1). No obstante es necesario confirmar estos resultados debido a que existió una leve influencia de la condición PL durante los sprints de atletismo (Gráfico 1) además sería oportuno aislar la disciplina del atletismo bajo las mismas condiciones de fatiga, para confirmar los efectos ergogénicos de la $\mathrm{Cr}$ sobre esta disciplina. Bajo esta línea de investigación existe la posibilidad de elaborar muchos estudios sobre $\mathrm{Cr} y$ rendimiento donde los participantes realicen protocolos experimentales que se asemejen más a las condiciones reales de un atleta en competición o entrenamiento, tal como lo sugirió Junh (1999).

\section{REFERENCIAS}

Aaserund, R., Gramvik, P., Olsen, S. R., y Jensen, J. (1998). Creatine supplementation delays onset of fatigue during repeated bouts of sprint running. Scandinavian Journal of Medicine and Science in Sports, 8, 247-251.

American College of Sports Medicine Roundtable. (2000). The physiological and health effect of oral creatine supplementation. Medicine and Science in Sports, 32(3), 706-717.

Balsom, P. D., Söderlund, K., Sjödin, B., y Ekblom, B. (1995). Skeletal muscle metabolism during short duration high-intensity exercise: influence of creatine supplementation. Acta Physiologica Scandinavica, 154, 303-310.

Burke, L. M., Pyne, D. B., y Telford, R. D. (1996). Effect of oral creatine supplementation on singleeffort sprint performance in elite swimmers. International Journal of Sport Nutrition, 6, 222233.

Casey, A., Constantin-Teodosiu, D., Howell, S., Hultman, E., y Greenhaff, P. L. (1996). Creatine ingestion favorably affects performance and muscle metabolism during maximal exercise in humans. American Journal of Physiology, 271(34), E31-E37.

Engelhardt, M., Neumann, G., Berbalk, A., y Reuter, I. (1998). Creatine supplementation in endurance sports. Medicine and Science in Sports and Exercise, 30(7), 1123-1129.

Giacomoni, M., Bernard, T., Gavarry, O., Altare, S., y Falgairette, G. (2000). Influence of the mestrual cycle phase and mestrual symptoms on maximal anaerobic performance. Medicine and Science in Sports and Exercise, 32(5), 486-492.

Green, A. L., Hultman, E., Macdonald, I. A., Sewell, D. A., y Greenhaff, P. L. (1996). Carbohydrate ingestion augments skeletal muscle creatine accumulation during creatine supplementation in 
humans. American Journal of Physiology, 271(34), E821-E826.

Green, J. M., McLester, J. R., Smith, J. F., y Mansfield, E. R. (2001). The effects of creatine supplementation on repeated upper- and lowerbody Wingate performance. Journal of Strength and Conditioning Research, 15(1), 36-41.

Greenhaff, P. L. (1995). Creatine and its application as an ergogenic aid. International Journal of Sport Nutrition, 5, S100-S110.

Greenhaff, P. L., Bodin, K., Soderlund, K., y Hultman, E. (1994). Effect of oral creatine supplementation on skeletal phosphocreatine resynthesis. American Journal of Phsiology, 266(29), E725-E730.

Grindstaff, P. D., Kreider, R., Bishop, R., Wilson, M., Wood, L., Alexander, C., y Almada, A. (1997). Effects of creatine supplementation on repetitive sprint performance and body composition in competitive swimmers. International Journal of Sport Nutrition, 7, 330-346.

Haff, G. G., Kirksey, K. B., Stone, M. H., Warren, B. J., Johnson, R. L., Stone, M., O'Bryant, H., y Proulx, C. (2000). The effect of 6 weeks of creatine monohydrate supplementation on dynamic rate of force development. Journal of Strength and Conditioning Research, 14(4), 426-433.

Harris, R. C., Viru, M., Greenhaff, P., y Hultman E. (1993). The effect of oral creatine supplementation on running performance during maximal short term exercise in man. Journal Physiology, 467, 74P.

Juhn, M. S. (1999). Oral creatine supplementation: separating fact from hype. The Physician and SportsMedicine, 27(5), 47-61.

Kamber, M., Koster, M., Kreis, R., Walker, G., Boesch, C., y Hoppeler, H. (1999). Creatine supplementation - Part I: performance, clinical chemistry, and muscle volume. Medicine and Science in Sports and Exercise, 31(12), 17631769.

Kreider, R. B., Ferreira, M., Wilson, M., Grindstaff, P., Plisk, S., Reinardy, J., Cantler, E., y Almada, A. L. (1998). Effects of creatine supplementation on body composition, strength, and sprint performance. Medicine and Science in Sports and Exercise, 30(1), 73-82.

Kreis, R., Kamber, M., Koster, M., Felblinger, J., Slotboom, J., Hoppeler, H., y Boesch, C. (1999). Creatine supplementation - Part II: in vivo magnetic resonance spectroscopy. Medicine and Science in Sports and Exercise, 31(12), 17701777.

Lamb, D. (1999). Judging the creatine effects on sport performance: 2.0 or 9.9?. En Memoria del VI Simposio Internacional de Ciencias del Deporte, el Ejercicio y la Salud. San José, Costa Rica: Universidad de Costa Rica.

McNaughton, L. R., Dalton, B., Tarr, J. (1998). The effects of creatine supplementation on hingintensity exercise performance in elite performer.
European Journal Appling Physiology, 78(3), 236-240.

Peyrebrune, M. C., Nevill, M. E., Donaldson, F. J., y Cosford, D. J. (1998). The effects of oral creatine supplementation on performance in single and repeated sprint swimming. Journal of Sport Sciences, 16, 271-279.

Poortmans, R., J., y Francaux, M. (2000). Adverse Effects of creatine supplementation: Fact or Fiction?. Sports Medicine, 30(3), 155-170.

Preen, D., Dawson, B., Goodman, C., Lawrence, S., Beilby, J., y Ching, S. (2001). Effect of creatine loading on long-term sprint exercise performance and metabolism. Medicine and Science in Sports and Exercise, 33(5), 814-821.

Prevost, M. C., Nelson, A. G., y Morris, G. S. (1997). Creatine supplementation enhances intermittent work performance. Research Quarterly for Exercise and Sports, 68(3), 233-240.

Redondo, D. R., Dowling, E. A., Graham, B. L., Almada, A. L., y Williams, M. H. (1996). The effect of oral creatine monohydrate supplementation on running velocity. International Journal of Sport Nutrition, 6, 213 221.

Rockwell, J. A., Walberg-Rankin, J., y Toderico, B. (2001). Creatine supplementation affects muscle creatine energy restriction. Medicine and Science in Sports and Exercise, 33(1), 61-68.

Rossiter, H. B., Cannell, E. R., y Jakeman, P. M. (1996). The effect of oral creatine supplementation on the 1000-m performance of competitive rowers. Journal of Sports Sciences, 14, 175-179.

Smith, S. A., Montain, S. J., Matott, R. P., Zientara, G. P., Jolesz, F. A., y Fielding, R. A. (1998). Creatine supplementation and age influence muscle metabolism during exercise. Journal of Applied Physiology, 85(4), 1349-1356.

Snow, R. J., McKenna, S. E., Kemp, J., Stathis, C. G., y Zhao, S. (1998). Effect of creatine supplementation on sprint exercise performance and muscle metabolism. Journal of Applied Physiology, 84(5), 1667-1673.

Statistical Package for Social Sciences (SPSS) (1998). SPSS advanced statistics 8.0. Chicago, IL: SPSS.

Terrillion, K. A., Kolkhorst, F. W., Dolgener, F. A., y Joslyn, S. J. (1997). The effect of creatine supplementation on two 700-m maximal running bouts. International Journal of Sport Nutrition, 7, 138-143.

Volek, J. S., Duncan, N. D., Mazzetti, S. A., Staron, R. S., Putukian, M., Gómez, A. L., Pearson, D., Fink, W. J., y Kraemer, W. J. (1999). Performance and muscle fiber adaptations to creatine supplementation and heavy resistance training. Medicine and Science in Sports and Exercise, 31(8), 1147-1156. 